\title{
Parity-Affected Persistent Currents in Superconducting Nanorings
}

\author{
Sergei V. Sharov ${ }^{1,3}$ and Andrei D. Zaikin ${ }^{2,4}$ \\ ${ }^{1}$ Institut für Theoretische Festkörperphysik, Universität Karlsruhe, 76128 Karlsruhe, Germany \\ ${ }^{2}$ Forschungszentrum Karlsruhe, Institut für Nanotechnologie, 76021 Karlsruhe, Germany \\ ${ }^{3}$ Institute for Physics of Microstructures, Russian Academy of Sciences, 603950 Nizhny Novgorod, Russia \\ ${ }^{4}$ I.E.Tamm Department of Theoretical Physics, P.N.Lebedev Physics Institute, 119991 Moscow, Russia
}

(Dated: November 23, 2018)

\begin{abstract}
We argue that at sufficiently low temperatures $T$ superconducting parity effect may strongly influence equilibrium persistent currents $(\mathrm{PC})$ in isolated superconducting nanorings containing a weak link with few conducting modes. An odd electron, being added to the ring, occupies the lowest available Andreev state and produces a countercurrent circulating inside the ring. For a single channel quantum point contact at $T=0$ this countercurrent exactly compensates the supercurrent $I_{e}$ produced by all other electrons and, hence, yields complete blocking of $\mathrm{PC}$ for any value of the external magnetic flux. In superconducting nanorings with embedded normal metal the odd electron countercurrent can "overcompensate" $I_{e}$ and a novel " $\pi / N$-junction" state occurs in the system. Changing the electron parity number from even to odd results in spontaneous supercurrent in the ground state of such rings without any externally applied magnetic flux.
\end{abstract}

PACS numbers: 74.78.Na, 73.23.Ra, 74.45.+c, 74.50.+r

Thermodynamic properties of isolated superconducting systems are sensitive to the parity of the total number of electrons [1, 2] even though this number $\mathcal{N}$ is macroscopically large. This parity effect is a fundamental property of a superconducting ground state described by the condensate of Cooper pairs. The number of electrons in the condensate is necessarily even, hence, for odd $\mathcal{N}$ at least one electron remains unpaired having an extra energy equal to the superconducting energy gap $\Delta$. This effect makes thermodynamic properties of the ground states with even and odd $\mathcal{N}$ differ. Clear evidence for such parity effect was demonstrated experimentally in small superconducting islands [2, 3].

Can the supercurrent be affected by this parity effect? In many structures the answer to this question is negative because of the fundamental uncertainty relation $\delta \mathcal{N} \delta \varphi \gtrsim 1$. Should the electron number $\mathcal{N}$ be fixed, fluctuations of the superconducting phase $\varphi$ become large disrupting the supercurrent. On the other hand, in transport experiments with fluctuations of $\varphi$ being suppressed the parity effect cannot be observed because of large fluctuations of $\mathcal{N}$.

Nonetheless, the parity effect can coexist with nonvanishing supercurrent. The way out is to consider systems supporting circular persistent currents (PC), such as, e.g., isolated superconducting rings pierced by the magnetic flux $\Phi$. In accordance with the number-phase uncertainty relation the global superconducting phase of the ring fluctuates strongly in this case, however these fluctuations are decoupled from the supercurrent and therefore cannot influence the latter. The aim of this paper is to demonstrate that the parity effect may substantially modify PC in superconducting nanorings, in particular if the total number of electrons is odd.

Consider, for instance, a single mode quantum point contact (QPC) embedded in a superconducting ring.
Provided the number of electrons in the system is not fixed the Josephson current across this contact is given by the well known expression [4]

$$
I(\varphi)=-\frac{2 e}{\hbar} \frac{\partial \varepsilon(\varphi)}{\partial \varphi} \tanh \frac{\varepsilon(\varphi)}{2 T}
$$

where $\varphi$ is the phase difference across QPC, $\varepsilon(\varphi)=$ $\Delta \sqrt{1-\mathcal{T} \sin ^{2}(\varphi / 2)}$ and $\mathcal{T}$ is the contact transmission. This result has a very transparent physical interpretation being related [5] to contributions of discrete Andreev energy states $E_{ \pm}= \pm \epsilon(\varphi)$ inside QPC via

$$
I(\varphi)=\frac{2 e}{\hbar}\left[\frac{\partial E_{-}}{\partial \varphi} f_{-}\left(E_{-}\right)+\frac{\partial E_{+}}{\partial \varphi} f_{+}\left(E_{+}\right)\right] .
$$

Using the Fermi filling factors for these states $f_{ \pm}\left(E_{ \pm}\right)=$ $[1+\exp ( \pm \epsilon(\varphi) / T)]^{-1}$ one arrives at Eq. (1).

Let us now fix the number of electrons inside the ring and consider the limit $T \rightarrow 0$. For even $\mathcal{N}$ all electrons are paired occupying available states with energies below the Fermi level (see Fig. 1a). In this case one has a

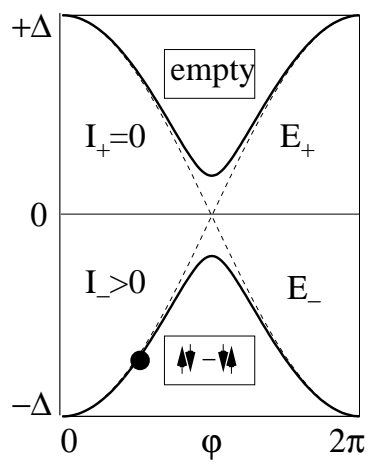

$\mathrm{b}$

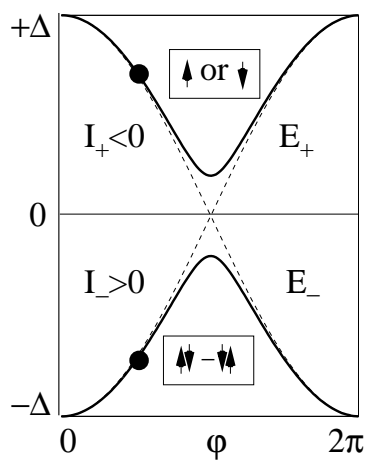

FIG. 1: Andreev levels inside QPC and their occupation at $T=0$ for even (a) and odd (b) ensembles. 
$f_{-}\left(E_{-}\right)=1, f_{+}\left(E_{+}\right)=0$, the current is entirely determined by the contribution of the quasiparticle state $E_{-}$ and Eq. (2) yields the same result as one for the grand canonical ensemble. By contrast, in the case of odd $\mathcal{N}$ one electron always remains unpaired and occupies the lowest available energy state - in our case $E_{+}$- above the Fermi level. Hence, for odd $\mathcal{N}$ one has $f_{ \pm}\left(E_{ \pm}\right)=1$ (Fig. 1b), the contributions of the two Andreev states in Eq. (2) exactly cancel each other, and the current across QPC remains zero for any $\varphi$ or the magnetic flux $\Phi$. Thus, we predict a novel mesoscopic effect - parity-induced blocking of $\mathrm{PC}$ in superconducting nanorings with embedded QPC [6].

It is important to emphasize that the above physical picture is based on the assumption that the supercurrent is determined only by the contributions of two discrete Andreev levels $E_{ \pm}(\varphi)$. This is the case provided (a) quasiparticle states of the continuum do not contribute to the supercurrent and (b) there are no more discrete Andreev levels inside the junction. Both these conditions are met only for symmetric and extremely short QPC. In a general case of $S N S$ junctions with a non-vanishing thickness of the normal layer $d \neq 0$ these conditions are violated, and no exact compensation of the supercurrent by the odd electron countercurrent is anymore possible.

Further analysis reveals even richer physics in the latter case. In particular, below we will demonstrate that at low $T$ and for odd number of electrons a novel $\pi / N$ junction state should occur in such $S N S$ rings. This effect in turn leads to spontaneous supercurrent flowing in the ring in its ground state. Under certain conditions the magnitude of this current can be as high as $I_{s p} \sim e \Delta / \hbar$ reaching the values up to $10 \div 100 \mathrm{nA}$ for generic BCS superconductors. Spontaneous currents of this magnitude can reliably be detected in modern experiments.

Parity projection formalism. In order to systematically investigate an interplay between the parity effect and PC in superconducting nanorings we will employ the parity projection formalism 7, 8, 9].

The grand canonical partition function $\mathcal{Z}(T, \mu)=$ $\operatorname{Tr} e^{-\beta(\mathcal{H}-\mu \mathcal{N})}$ is linked to the canonical one $Z(T, \mathcal{N})$ as

$$
\mathcal{Z}(T, \mu)=\sum_{\mathcal{N}=0}^{\infty} Z(T, \mathcal{N}) \exp \left(\frac{\mu \mathcal{N}}{T}\right) .
$$

Here and below $\mathcal{H}$ is the system Hamiltonian and $\beta \equiv$ $1 / T$. Inverting this relation and defining the canonical partition functions $Z_{e}$ and $Z_{o}$ respectively for even $(\mathcal{N} \equiv$ $\left.\mathcal{N}_{e}\right)$ and odd $\left(\mathcal{N} \equiv \mathcal{N}_{o}\right)$ ensembles, one gets

$$
Z_{e / o}(T)=\frac{1}{2 \pi} \int_{-\pi}^{\pi} d u e^{-i \mathcal{N}_{e / o} u} \mathcal{Z}_{e / o}(T, i T u),
$$

where

$$
\mathcal{Z}_{e / o}(T, \mu)=\frac{1}{2} \operatorname{Tr}\left\{\left[1 \pm(-1)^{\mathcal{N}}\right] e^{-\beta(\mathcal{H}-\mu \mathcal{N})}\right\}
$$

$$
=\frac{1}{2}(\mathcal{Z}(T, \mu) \pm \mathcal{Z}(T, \mu+i \pi T))
$$

are the parity projected grand canonical partition functions. For $\mathcal{N} \gg 1$ it is sufficient to evaluate the integral in (4) within the saddle point approximation

$$
Z_{e / o}(T) \sim e^{-\beta\left(\Omega_{e / o}-\mu_{e / o} \mathcal{N}_{e / o}\right)},
$$

where $\Omega_{e / o}=-T \ln \mathcal{Z}_{e / o}(T, \mu)$ are the parity projected thermodynamic potentials,

$$
\Omega_{e / o}=\Omega_{f}-T \ln \left[\frac{1}{2}\left(1 \pm e^{-\beta\left(\Omega_{b}-\Omega_{f}\right)}\right)\right]
$$

and $\Omega_{f / b}=-T \ln \left[\operatorname{Tr}\left\{( \pm 1)^{\mathcal{N}} e^{-\beta(\mathcal{H}-\mu \mathcal{N})}\right\}\right]$. "Chemical potentials" $\mu_{e / o}$ are defined by the saddle point condition $\mathcal{N}_{e / o}=-\partial \Omega_{e / o}\left(T, \mu_{e / o}\right) / \partial \mu_{e / o}$.

The main advantage of the above formalism is that it allows to express the canonical partition functions and thermodynamical potentials in terms of the parity projected grand canonical ones thereby enormously simplifying the whole calculation. We further note that $\Omega_{f}$ is just the standard grand canonical thermodynamic potential and $\Omega_{b}$ represents the corresponding potential linked to the partition function $\mathcal{Z}(T, \mu+i \pi T)$. It is easy to see 8] that in order to recover this function one can evaluate the true grand canonical partition function $\mathcal{Z}(T, \mu)$, express the result as a sum over the Fermi Matsubara frequencies $\omega_{f}=2 \pi T(l+1 / 2)$ and then substitute the Bose Matsubara frequencies $\omega_{b}=2 \pi T l(l=0, \pm 1, \ldots)$ instead of $\omega_{f}$. This procedure automatically yields the correct expression for $\mathcal{Z}(T, \mu+i \pi T)$ and, hence, for $\Omega_{b}$.

Having found the thermodynamic potentials for the even and odd ensembles one can easily determine the equilibrium current $I_{e / o}$. Consider, as before, isolated superconducting rings pierced by the magnetic flux $\Phi$. Making use of the above expressions one finds PC circulating inside the ring:

$$
I_{e / o}=I_{f} \pm \frac{I_{b}-I_{f}}{e^{\beta\left(\Omega_{b}-\Omega_{f}\right)} \pm 1},
$$

where the upper/lower sign corresponds to the even/odd ensemble and we have defined

$$
I_{e / o}=-c\left(\frac{\partial \Omega_{e / o}}{\partial \Phi}\right)_{\mu(\Phi)}, \quad I_{f / b}=-c\left(\frac{\partial \Omega_{f / b}}{\partial \Phi}\right)_{\mu(\Phi)} .
$$

Homogeneous superconducting rings. Turning to concrete calculations we first treat homogeneous nanorings with cross section $s$ and perimeter $L=2 \pi R$. Throughout this paper rings will be assumed sufficiently thin, $\sqrt{s}<\lambda_{L}$, where $\lambda_{L}$ is the London penetration length. Superconducting properties of such rings will be described within the (parity projected) mean field BCS theory. At low temperatures this description is justified provided quantum fluctuations of the order parameter, quantum phase slips (QPS) 10], can be neglected. This requirement can 
be fulfilled only provided the total number of conducting channels in the ring remains large [1] $N_{\mathrm{r}} \sim p_{F}^{2} s \gg 1$. In addition, the perimeter $L$ should not be too large in order to disregard the QPS-induced reduction of PC 13 . Finally, we will neglect the difference between the mean field values of the BCS order parameter for the even and odd ensembles [7, 8]. This is legitimate provided the ring volume is large enough, $\mathcal{V}=L s \gg 1 / \nu \Delta$, where $\nu$ is the density of states at the Fermi level and $\Delta$ is the BCS order parameter for a bulk superconductor at $T=0$.

The task at hand is now to evaluate the thermodynamic potentials $\Omega_{f / b}$. Within the mean field approach these quantities can be expressed in terms of the excitation energies $\varepsilon_{k}$ and the order parameter $\Delta(\boldsymbol{r})$. One finds $[8]$

$$
\begin{aligned}
& \Omega_{f}=\tilde{\Omega}-2 T \sum_{k} \ln \left(2 \cosh \frac{\varepsilon_{k}}{2 T}\right), \\
& \Omega_{b}=\tilde{\Omega}-2 T \sum_{k} \ln \left(2 \sinh \frac{\varepsilon_{k}}{2 T}\right),
\end{aligned}
$$

where $\tilde{\Omega}=\int d^{3} \boldsymbol{r}|\Delta(\boldsymbol{r})|^{2} / g+\operatorname{Tr}\{\hat{\xi}\}, g$ is the BCS coupling constant and $\hat{\xi}$ is the single-particle energy operator:

$$
\hat{\xi}=\frac{1}{2 m}\left(-i \hbar \frac{\partial}{\partial \boldsymbol{r}}-\frac{e}{c} \boldsymbol{A}(\boldsymbol{r})\right)^{2}+U(\boldsymbol{r})-\mu,
$$

$\boldsymbol{A}(\boldsymbol{r})$ is the vector potential and $U(\boldsymbol{r})$ describes the potential profile due to disorder and interfaces.

The excitation spectrum $\varepsilon_{k}$ has the form

$$
\varepsilon_{k}=\varepsilon(\boldsymbol{p})=\boldsymbol{p} \boldsymbol{v}_{S}+\sqrt{\xi^{2}+\Delta^{2}},
$$

where $\boldsymbol{p}$ is a quasiparticle momentum, $\xi=\left(p^{2}-\tilde{\mu}\right) / 2 m$, $\tilde{\mu}=\mu(\Phi)-m v_{S}^{2} / 2$ and

$$
v_{S}=\frac{\hbar}{2 m R} \min _{n}\left(n-\frac{\Phi}{\Phi_{0}}\right)
$$

is the superconducting velocity. Both $\varepsilon(\boldsymbol{p})$ and $v_{S}$ are periodic functions of the flux $\Phi$ with the period equal to the superconducting flux quantum $\Phi_{0}=h c / 2 e$.

Consider the most interesting case $T \ll \hbar v_{F} / L$ [14]. Making use of the above expressions one obtains

$$
I_{e}=e v_{S} \varrho_{e} s, \quad I_{o}=e v_{S} \varrho_{o} s-e \frac{v_{F}}{L} \operatorname{sgn} v_{S},
$$

where $\varrho_{e / o}=\mathcal{N}_{e / o} / \mathcal{V}$. The first Eq. (14) - together with (13) - coincides with that for the grand canonical ensemble. In contrast, for odd ensembles (second Eq. (14) ) there exists an additional flux-dependent term which, however, remains rather small in multichannel rings [15]. Estimating the main contribution to $I_{e / o}$ as $I \sim e v_{F} N_{\mathrm{r}} / L$, one gets $\left(I_{e}-I_{o}\right) / I \sim 1 / N_{\mathrm{r}} \ll 1$.

Now we proceed to the situations in which parity effect gains more importance and leads to new physical effects. Namely, we consider superconducting rings with

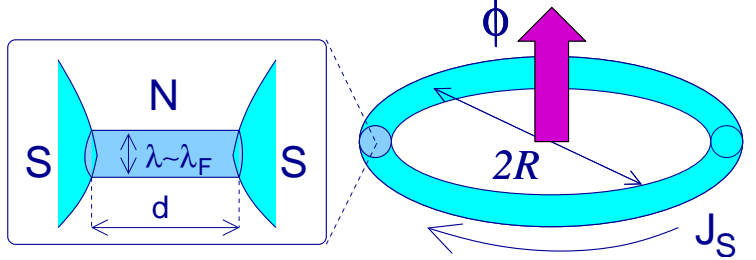

FIG. 2: Superconducting ring with embedded $S N S$ junction of length $d$.

$N_{\mathrm{r}} \gg 1$ interrupted by a weak link with only few conducting channels $N$. In such systems the mean field BCS description remains applicable and, on the other hand, the parity effect can be large due to the condition $N \sim 1$. Quantum point contacts (QPC) or, more generally, $S N S$ junctions (of length $d$ ) can be used for practical realization of such weak links. The corresponding structure is depicted in Fig. 2.

Superconducting rings with $Q P C$. As a first example we consider a ring interrupted by QPC. The optimal value of the phase difference $\varphi$ across QPC is fixed by the equation $\partial \Omega_{e / o} / \partial \varphi=0$, which yields the current conservation condition $I^{(c)}=I^{(r)}$. Here $I^{(c)} \sim 2 e \mathcal{T} \Delta / \hbar$ is the current through the contact and $I^{(r)} \simeq\left(e v_{F} N_{\mathrm{r}} / L\right)\left(\varphi-2 \pi \Phi / \Phi_{0}\right)$ is the supercurrent inside the ring. From the current conservation one easily finds [16]

$$
\begin{array}{ll}
\varphi \simeq 2 \pi \Phi / \Phi_{0}, & \text { if } \quad L \ll L^{*}, \\
\varphi \simeq 2 \pi n, & \text { if } L \gg L^{*},
\end{array}
$$

where $L^{*}=\xi_{0} N_{\mathrm{r}} / \mathcal{T} \gg \xi_{0} \sim \hbar v_{F} / \Delta$. In a more general case of QPC with $N$ conducting channels in the expression for $L^{*}$ one should set $\mathcal{T} \rightarrow \sum_{i}^{N} \mathcal{T}_{i}$.

In the limit (16) one recovers the results identical to ones for homogeneous rings. Below we will concentrate on the opposite limit $L \ll L^{*}$ and also assume $N \ll N_{\mathrm{r}}$. Due to Eq. (15) in this case the dependence $I_{e / o}(\Phi)$ is fully determined by the current-phase relation for QPC which can be found by means of Eq. (8). Expressing $I_{f / b}$ via $\varphi$ it is convenient to employ the general formula [17]

$$
I_{f / b}=\frac{2 e}{\hbar} \sum_{i=1}^{N} T \sum_{\omega_{f / b}} \frac{\sin \varphi}{\cos \varphi+W_{i}\left(\omega_{f / b}\right)} .
$$

For QPC one has $W_{i}(\omega)=\left(2 / \mathcal{T}_{i}\right)\left(1+\hbar^{2} \omega^{2} / \Delta^{2}\right)-1$. Substituting this function into (17) and summing over $\omega_{f}$ one rederives the standard result [4], while the same summation over $\omega_{b}$ yields

$$
I_{b}=-\frac{2 e}{\hbar} \sum_{i=1}^{N} \frac{\partial \varepsilon_{i}(\varphi)}{\partial \varphi} \operatorname{coth} \frac{\varepsilon_{i}(\varphi)}{2 T},
$$

where $\varepsilon_{i}(\varphi)=\Delta \sqrt{1-\mathcal{T}_{i} \sin ^{2}(\varphi / 2)}$. Finally, the difference $\Omega_{b}-\Omega_{f} \equiv \Omega_{b f}$ is evaluated as a sum of the ring 
$\left(\Omega_{b f}^{(r)}\right)$ and $\mathrm{QPC}\left(\Omega_{b f}^{(c)}\right)$ contributions. The latter is found by integrating $I_{f / b}(\varphi)$ over the phase $\varphi$ with the result

$$
\Omega_{b f}^{(c)}=2 T \sum_{i=1}^{N} \ln \operatorname{coth}\left(\frac{\varepsilon_{i}(\varphi)}{2 T}\right),
$$

while the former is defined by the standard expression

$$
\beta \Omega_{b f}^{(r)}=2 \mathcal{V} \int \frac{d^{3} \boldsymbol{p}}{(2 \pi \hbar)^{3}} \ln \left(\operatorname{coth} \frac{\varepsilon(\boldsymbol{p})}{2 T}\right) \simeq \nu \mathcal{V} \sqrt{\Delta T} e^{-\frac{\Delta}{T}}
$$

Combining all these results with Eq. (8) we get

$$
\begin{gathered}
I_{e / o}=-\frac{2 e}{\hbar} \sum_{i=1}^{N} \frac{\partial \varepsilon_{i}(\varphi)}{\partial \varphi} \tanh \frac{\varepsilon_{i}(\varphi)}{2 T} \\
\times\left[1 \pm \frac{\left(\operatorname{coth} \frac{\varepsilon_{i}(\varphi)}{2 T}\right)^{2}-1}{e^{\beta \Omega_{b f}^{(r)}} \prod_{j=1}^{N}\left(\operatorname{coth} \frac{\varepsilon_{j}(\varphi)}{2 T}\right)^{2} \pm 1}\right] .
\end{gathered}
$$

The term in the square brackets accounts for the parity effect in our system. For $N=1$ and at $T=0$ this term reduces to unity for even ensembles and to zero for odd ones, thus confirming our intuitive picture of parityinduced blocking of PC discussed above. For $T>0$ Eq. (20) demonstrates that both for even and especially for odd $\mathcal{N}$ the current-phase relation for QPC may substantially deviate from that derived for the grand canonical ensemble [4], see Fig. 3. For even ensembles the supercurrent increases above its grand canonical value. This effect is mainly pronounced for phases $\varphi$ not very far

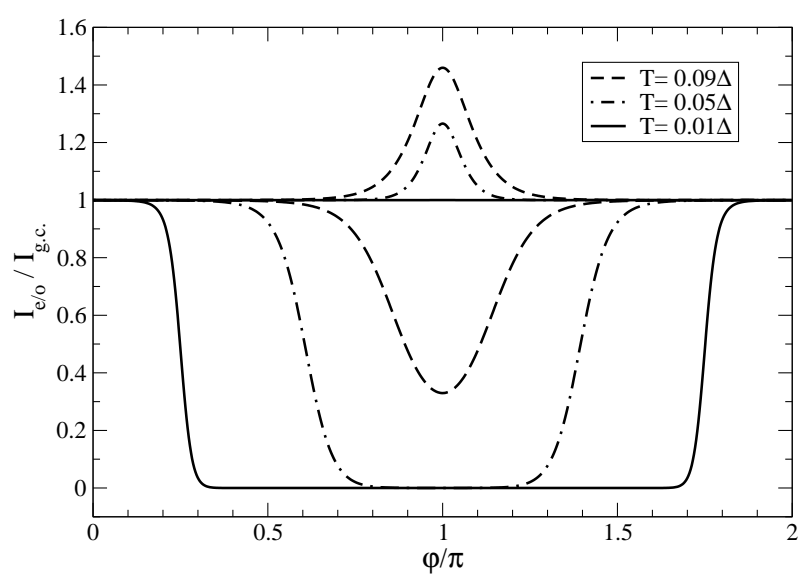

FIG. 3: The ratio between canonical and grand canonical values of PC $I_{e / o} / I_{g . c .}$ (represented by the term in the square brackets in Eq. (20) versus $\varphi$ in a single mode QPC at different temperatures for even (three upper curves) and odd (three lower curves) ensembles. Here we have chosen $T_{S}^{*}=0.1 \Delta$ and $\mathcal{T}=0.99$. from $\varphi=\pi$ and - at sufficiently low $T$ - it becomes progressively more important with increasing temperature. On the contrary, for odd ensembles the supercurrent is always suppressed below its grand canonical value. This suppression is gradually lifted with increasing temperature, though at phases $\varphi$ in the vicinity of the point $\varphi=\pi$ blocking of PC may persist up to sufficiently high $T$. Eq. (20) also shows that in QPC with several conducting channels and at $T \rightarrow 0$ the current through the most transparent channel will be blocked by the odd electron. Hence, though blocking of PC remains incomplete in this case, it may nevertheless be important also for QPC with $N>1$.

SNS rings. Finally, we turn to superconducting rings containing a piece of a normal metal with non-zero thickness $d$. In contrast to the situation of QPC considered above, the Josephson current in $S N S$ structures cannot anymore be attributed only to the discrete Andreev states inside a weak link, and an additional contribution from the states in the continuum should also be taken into account. Furthermore, for any non-zero $d$ there are always more than two discrete Andreev levels in the system. Accordingly, significant modifications in the physical picture of the parity effect in $S N S$ rings can be expected.

The key difference can be understood already by comparing the typical structure of discrete Andreev levels in $S N S$ junctions (Fig. 4) with that of QPC (Fig. 1). As before, in the limit $T \rightarrow 0$ all states below (above) the Fermi level are occupied (empty) provided the total number of electrons in the system is even (Fig. 4a). If, on the other hand, this number is odd the lowest Andreev state above the Fermi energy is occupied as well (Fig. 4b) thus providing an additional contribution to the Josephson current. This contribution, however, cancels only that of a symmetric Andreev level below the Fermi energy, while the contributions of all other occupied Andreev levels and of the continuum states remain uncompensated. Hence, unlike in the QPC limit displayed in Fig. 1, in $S N S$ rings
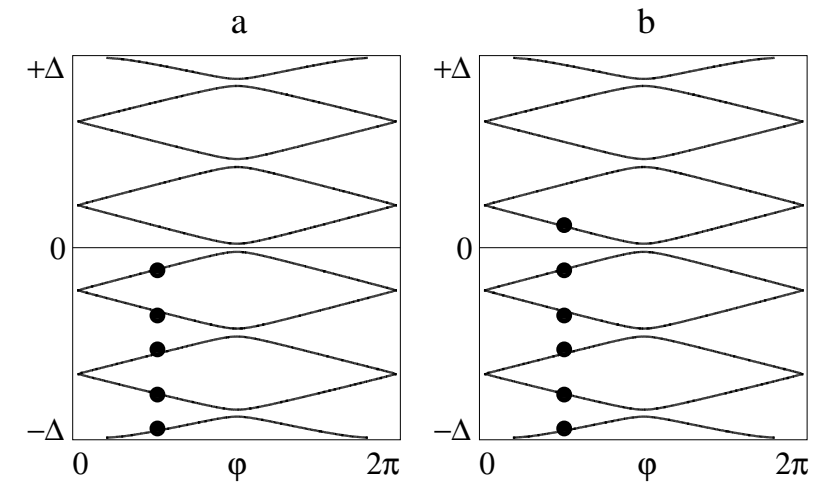

FIG. 4: Andreev levels in a single mode $S N S$ junction with $d=6 \hbar v_{F} / \Delta$ and their occupation at $T=0$ for even (a) and odd (b) ensembles. 
one should not anymore expect the effect of PC blocking by the odd electron, but rather some other non-trivial features of the parity effect.

In order to construct a quantitative description of the parity effect in $S N S$ rings we will again make use of the parity projection technique formulated above. Here we only restrict our attention to transparent $S N S$ junctions where the effects under consideration are most pronounced. In this case the function $W_{i}(\omega) \equiv W(\omega)$ is the same for all channels. It reads

$$
\begin{gathered}
W(\omega)=\left(\frac{2 \hbar^{2} \omega^{2}}{\Delta^{2}}+1\right) \cosh \left(\frac{2 \omega d}{v_{F}}\right) \\
+\frac{2 \hbar \omega}{\Delta} \sqrt{1+\frac{\hbar^{2} \omega^{2}}{\Delta^{2}}} \sinh \left(\frac{2 \omega d}{v_{F}}\right) .
\end{gathered}
$$

Substituting this function into (17) and repeating the whole calculation as above, we arrive at the final result which cannot in general be represented in a tractable analytic form. Significant simplifications occur in the most interesting limit [18] $T \rightarrow 0$, in which case we obtain

$$
\begin{aligned}
& I_{e}=\frac{e \Delta N}{\hbar}\left(\sin \frac{\varphi}{2}-\frac{2 y \sin \varphi}{\pi} \ln \frac{1}{y}\right), \\
& I_{o}=I_{e}-\frac{e \Delta}{\hbar}\left(\sin \frac{\varphi}{2}+y \operatorname{sgn} \varphi \cos \varphi\right)
\end{aligned}
$$

for short $S N S$ junctions $y \equiv d \Delta / \hbar v_{F} \ll 1$ and

$$
I_{e}=\frac{e v_{F} N}{\pi d} \varphi, \quad I_{o}=\frac{e v_{F} N}{\pi d}\left(\varphi-\frac{\pi \operatorname{sgn} \varphi}{N}\right)
$$

for long ones $d \gg \xi_{0} \sim \hbar v_{F} / \Delta$. These results apply for $-\pi<\varphi<\pi$ and should be $2 \pi$-periodically continued otherwise. The term containing $\ln (1 / y)$ in Eq. (23) for $I_{e}$ is written with the logarithmic accuracy and is valid for $\varphi$ not too close to $\varphi= \pm \pi$.

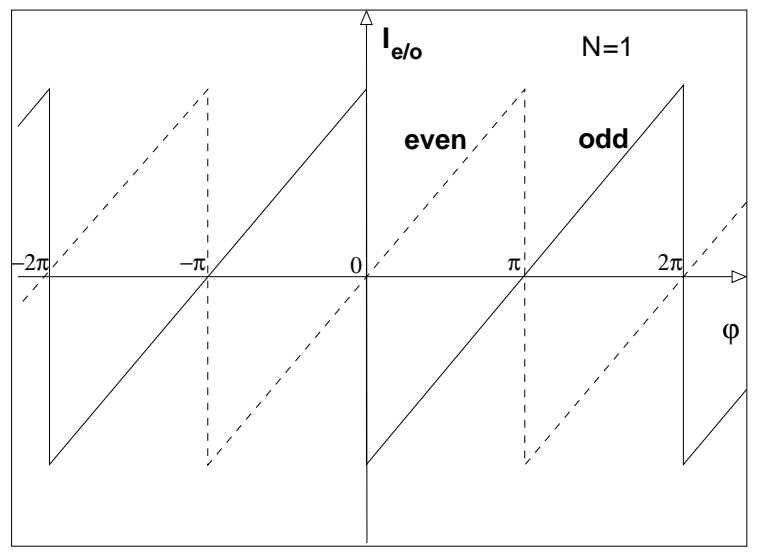

FIG. 5: The zero temperature current-phase dependence (24) for $S N S$ rings with $N=1: I_{e}(\varphi)$ (dashed) line and $I_{o}(\varphi)$ (solid) line.
Let us concentrate on the results (24) applicable for sufficiently long $S N S$ junctions. We observe that at $T=0$ the current $I_{e}$ - as in the QPC case - coincides with that for the grand canonical ensembles 19], while for odd $\mathcal{N}$ the current-phase relation is shifted by the value $\pi / N$. This shift has a simple interpretation being related to the odd electron contribution $(2 e / \hbar) \partial E_{1} / \partial \varphi$ from the lowest (above the Fermi level) Andreev state $E_{1}(\varphi)$ inside the $S N S$ junction. As we have expected, this contribution indeed does not compensate for the current from other quasiparticle states. Rather it provides a possibility for a parity-induced $\pi$-junction state [20] in our system: According to Eq. (24) for single mode $S N S$ junctions the "saw tooth" current-phase relation will be shifted exactly by $\pi$, see Fig. 5 . More generally, we can talk about a novel $\pi / N$-junction state, because in the odd case the minimum Josephson energy (zero current) state is reached at $\varphi= \pm \pi / N$, see Fig. 6. For any $N>1$ this is a twofold degenerate state within the interval $-\pi<\varphi<\pi$ 21]. In the particular case $N=2$ the current-phase relation $I_{o}(\varphi)$ turns $\pi$-periodic.

Let us recall that the $\pi$-junction state can be realized in $S N S$ structures by driving the electron distribution function in the contact area out of equilibrium 22, 23, 24]. Here, in contrast, the situation of a $\pi$ - or $\pi / N$-junction is achieved in thermodynamic equilibrium. Despite this drastic difference, there also exists a certain physical similarity between the effects discussed here and in Refs. 22, 23, 24: In both cases the electron distribution function in the weak link deviates substantially from the Fermi function. It is this deviation which is responsible for the appearance of the $\pi$-junction state in both physical situations.

It is also important to emphasize that the countercurrent produced by the odd electron causes a jump on the current-phase dependence at $\varphi=0$, and the direction of $I_{o}$ at sufficiently small $\varphi$ is always opposite to that of $I_{e}$. This is a generic feature of odd ensembles which

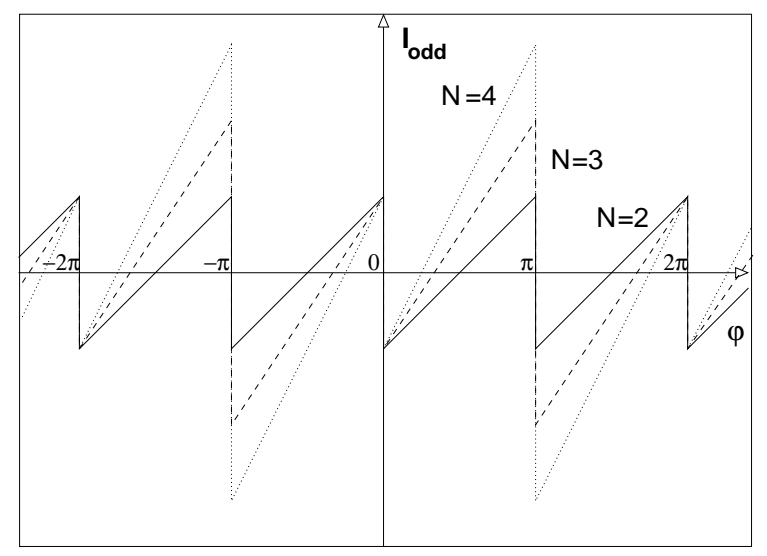

FIG. 6: The same as in Fig. 5 only for the odd ensembles (second Eq. (24)) and for $N=2,3$ and 4 . 


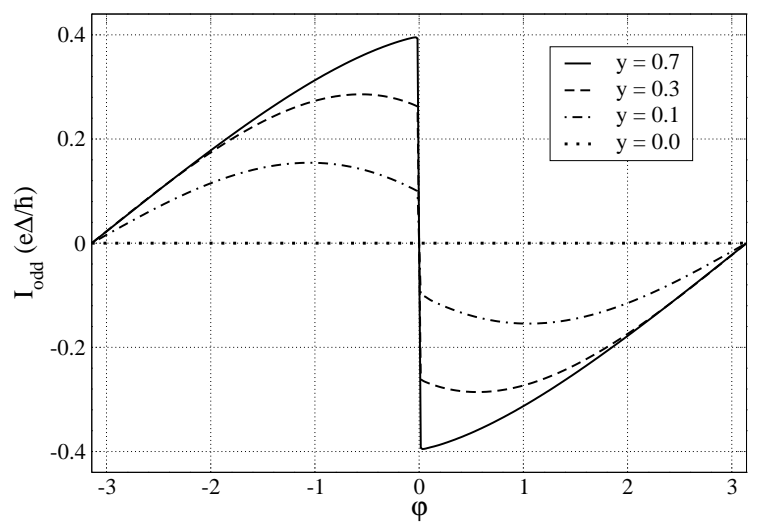

FIG. 7: The zero temperature current-phase relation $I_{o}(\varphi)$ $(-\pi<\varphi<\pi)$ for $N=1$ and different values of the parameter $y=d \Delta / \hbar v_{F}$.

persists for all non-zero values of $y$. The current-phase relation $I_{o}(\varphi)$ for arbitrary values of the parameter $y$ can be computed numerically. Examples are presented in Fig. 7. One clearly observes the current jump at $\varphi=0$. As it is obvious from Eq. (23), this feature disappears only in the QPC limit $y \rightarrow 0$.

$B C S$ ground state with spontaneous current. Perhaps the most spectacular physical consequence of this current jump is the presence of spontaneous supercurrents in the ground state of $S N S$ rings with odd number of electrons. Similarly to the case of standard $\pi$-junctions 20] such spontaneous supercurrents should flow even in the absence of an externally applied magnetic flux. Unlike in Ref. 20, however, here the spontaneous current state occurs for any inductance of the ring because of the non-sinusoidal dependence $I_{o}(\varphi)$.

Consider, for instance, the limit $d \gg \xi_{0}$. In the case of odd number of electrons the ground state energy of an $S N S$ ring can be written in a simple form

$$
E=\frac{\Phi^{2}}{2 c \mathcal{L}}+\frac{\pi \hbar v_{F} N}{\Phi_{0}^{2} d}\left(\Phi-\frac{\Phi_{0} \operatorname{sgn} \Phi}{2 N}\right)^{2}
$$

where the first term is the magnetic energy of the ring $(\mathcal{L}$ is the ring inductance and $c$ is the speed of light) while the second term represents the Josephson energy of the $S N S$ junction. Minimizing (25) with respect to the flux $\Phi$ one immediately concludes that the ground state of the ring is a twofold degenerate state with a non-vanishing spontaneous current

$$
I= \pm \frac{e v_{F}}{d}\left[1+\frac{2 e v_{F} N}{d} \frac{\mathcal{L}}{\Phi_{0}}\right]^{-1}
$$

flowing either clockwise or counterclockwise. In the limit of small inductances $\mathcal{L} \rightarrow 0$ this current does not vanish and its amplitude just reduces to that of the odd electron current at $\varphi \rightarrow 0$. Hence, at small ring inductances the

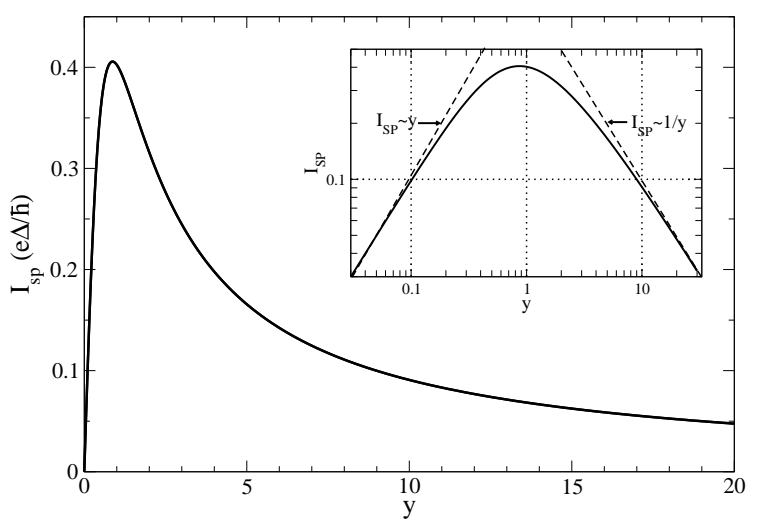

FIG. 8: The spontaneous current amplitude $I_{s p}$ as a function of the parameter $y$ at $T=0$. In the inset, the same function is shown on the $\log -\log$ scale. Dashed lines indicate the asymptotic behavior of $I_{s p}(y)$ in the limits of small and large $y$.

magnitude $I_{s p}$ of this spontaneous current can easily be obtained from Eqs. (2324):

$$
\begin{aligned}
I_{s p}=e \Delta^{2} d / \hbar^{2} v_{F}, & \text { if } d \ll \xi_{0}, \\
I_{s p}=e v_{F} / \pi d, & \text { if } d \gg \xi_{0} .
\end{aligned}
$$

For intermediate values of the parameter $y$ the amplitude of the current $I_{s p}$ can be evaluated numerically. The results are displayed in Fig. 8. One observes that - in agreement with Eq. (27) $-I_{s p}$ increases linearly with $d$ at small $d$, reaches its maximum value $I_{\max } \sim 0.4 e \Delta / \hbar$ at $d \sim \xi_{0}$ and then decreases with further increase of $d$ approaching the dependence (28) in the limit of large $d$. For generic BCS superconductors the magnitude of this maximum current can be estimated as $I_{\max } \sim 10 \div$ $100 \mathrm{nA}$. These values might be considered as surprisingly large ones having in mind that this current is associated with only one Andreev electron state.

In summary, new physical effects emerge from an interplay between the electron parity number and persistent currents in superconducting nanorings. Perhaps the most striking observation is that the BCS ground state of such rings with the odd number of electrons is the state with non-zero spontaneous supercurrent. This and other novel features predicted here can be directly tested in modern experiments.

This work is part of the Kompetenznetz "Funktionelle Nanostructuren" supported by the Landestiftung BadenWürttemberg gGmbH and of the STReP project "Ultra1D" supported by the EU.

[1] D.V. Averin and Yu.V. Nazarov, Phys. Rev. Lett. 69, 1993 (1992). 
[2] M.T. Tuominen et al., Phys. Rev. Lett. 69, 1997 (1992).

[3] P. Lafarge et al., Phys. Rev. Lett. 70, 994 (1993).

[4] I.O. Kulik and A.N. Omel'yanchuk, Sov. J. Low Temp. Phys. 4, 142 (1978); W. Haberkorn, H. Knauer, and J. Richter, Phys. Stat. Solidi (A) 47, K161 (1978).

[5] A. Furusaki and M. Tsukada, Physica B 165-166, 967 (1990); C.W.J. Beenakker and H. van Houten, Phys. Rev. Lett. 66, 3056 (1991).

[6] A similar proposal has recently been put forward by M. Hayashi and H. Ebisawa, Phys. Rev. B 67, 014524 (2003).

[7] B. Janko, A. Smith, and V. Ambegaokar, Phys. Rev. B 50, 1152 (1994).

[8] D.S. Golubev and A.D. Zaikin, Phys. Lett. A 195, 380 (1994).

[9] D.V. Averin and Yu.V. Nazarov, Physica B 203, 310 (1994).

[10] A.D. Zaikin et al., Phys. Rev. Lett. 78, 1552 (1997); D.S. Golubev and A.D. Zaikin, Phys. Rev. B 64, 014504 (2001).

[11] More detailed requirements can be formulated with the aid of the results [10]. For generic wires QPS effects are small provided the parameter $\sqrt{s}$ exceeds $\sim 10 \mathrm{~nm}[10$, 12].

[12] A. Bezryadin, C.N. Lau, and M. Tinkham, Nature (London) 404, 971 (2000); C.N. Lau et al., Phys. Rev. Lett. 87, 217003 (2001).

[13] K.A. Matveev, A.I. Larkin, and L.I. Glazman, Phys. Rev. Lett. 89, 096802 (2002).

[14] With increasing $T$ the parity effect gets weaker and eventually disappears at [2] $T>T_{S}^{*} \approx \Delta \ln \left(\nu \mathcal{V} \sqrt{\Delta T_{S}^{*}}\right)$.

[15] Within the framework of the mean field theory parity effect in single channel rings was discussed by K. Kang, Europhys. Lett. 51, 181 (2000) and by H.-J. Kwon and V.M. Yakovenko, Phys. Rev. Lett. 89, 017002 (2002). In such rings, however, QPS effects may entirely wipe out the supercurrent thus making the mean field approach obsolete.

[16] Quantum fluctuations of $\varphi$ can easily be suppressed, e.g. by choosing the ring inductance sufficiently small. Accordingly, the number of Cooper pairs passing through the contact should strongly fluctuate effectively suppressing electrostatic effects in the contact area.

[17] A.V. Galaktionov and A.D. Zaikin, Phys. Rev. B 65, 184507 (2002).

[18] As it was already pointed out, the parity effects persist at sufficiently low temperatures and disappear otherwise. For rings with sufficiently short weak links the corresponding crossover temperature is $T_{S}^{*}$ [14]. In the case of long $S N S$ junctions $d \gg \xi_{0}$ and for not very large $N$ the estimate of this crossover temperature is fully analogous and just amounts to calculating the number of states available for a quasiparticle at a given $T$. This estimate gives rise to the crossover temperature $T_{N}^{*} \sim \hbar v_{F} / d \ln \left(N T_{N}^{*} d / v_{F}\right)$ above which even-odd effects in long $S N S$ junctions should be smeared out. In a general case the crossover temperature is defined by either $T_{S}^{*}$ or $T_{N}^{*}$ whatever is smaller.

[19] I.O. Kulik, Sov. Phys. JETP 30, 944 (1970); C. Ishii, Progr. Theor. Phys. 44, 1525 (1970).

[20] L.N. Bulaevskii, V.V. Kuzii, and A.A. Sobyanin, JETP Lett. 25, 290 (1977).

[21] A similar behavior is expected for $S N S$ junctions formed by $d$-wave superconductors, for further discussion see Yu.S. Barash, A.V. Galaktionov and A.D. Zaikin, Phys. Rev. B 52, 665 (1995).

[22] A.F. Volkov, Phys. Rev. Lett. 74, 4730 (1995).

[23] F.K. Wilhelm, G. Schön, and A.D. Zaikin, Phys. Rev. Lett. 81, 1682 (1998).

[24] S.K. Yip, Phys. Rev. 58, 5803 (1998). 